\title{
Les marchands toscans face au latin vers 1400
}

\section{Indices de contacts linguistiques dans l'Archivio Datini}

\begin{abstract}
Il saggio propone una mappatura dei testi latini presenti nel più vasto fondo mercantile europeo, l'Archivio Datini di Prato, per verificarne la ripartizione differenziata secondo la tipologia dei documenti. Il latino non interviene praticamente nelle scritture di registrazione dei toscani e abbastanza poco nei saperi tecnici, testi religiosi e letterari collezionati da essi. Per la comunicazione, i mercanti toscani ricevono soprattutto lettere latine dai loro colleghi liguri e piemontesi e da religiosi e letterati, spesso stranieri. Tra i documenti giuridici, gli atti notarili e le procedure giudiziarie sono generalmente in latino. Mentre la lingua adottata è negoziata caso per caso tra mercanti di origini diverse attraverso vari modi di plurilinguismo, la mediazione dei notai si impone in modo quasi esclusivo per l'accesso alla lingua del diritto.
\end{abstract}

This contribution provides a typological categorization of the Latin texts preserved in the largest European collection of mercantile documents, the Archivio Datini in Prato. Latin is not used in the ledgers written in Tuscany, and hardly ever in the technical, religious, and literary writings collected there. However, the Tuscan merchants mainly receive Latin letters from their colleagues in Liguria and Piedmont, as well as from churchmen and scholars, often from abroad. Among the legal texts, notarial records and court procedures were generally written in Latin. While the language used in mercantile communication is negotiated case by case between merchants with different backgrounds, using various forms of bilingualism, notarial notaries are practically the only interpreters used to translate the language of law.

Parole chiave: Merchants, Pragmatic literacy, Vernacular Latin, Cultural mediation, Language of law

Dans le champ des études littéraires, la question de l'accès des marchands et d'autres illitterati de la période préhumaniste à une culture dont les formes supérieures étaient habituellement latines a surtout été pensée à travers le mou-

Jérôme Hayez, LAMOP - CNRS - Université Paris Sorbonne 
vement de traduction de textes religieux, littéraires et scientifiques anciens ou médiévaux (volgarizzamenti), qui se manifeste dans toute son ampleur à partir du XIV ${ }^{\mathrm{e}}$ siècle. ${ }^{1}$ Celui-ci ne constitue en réalité qu'un aspect parmi d'autres du rapport des marchands à des langues secondes. Les historiens se sont davantage intéressés à la communication entre négociants de nations différentes, avec les formes d'apprentissage, d'intercompréhension ou de traduction qu'elle impliquait dans le milieu cosmopolite des places commerciales. ${ }^{2}$ En parallèle, une évolution récente des études philologiques d'écrits marchands, de la lexicographie et de la caractérisation des dialectes anciens vers l'étude de phénomènes d'hybridation, a aussi focalisé son attention sur l'écriture de vernaculaires différents de la langue maternelle. ${ }^{3}$

\section{Marchands et contacts linguistiques}

L'étude de dossiers choisis d'écrits marchands, souvent composés de petites séries de missives, illustre habituellement l'interaction entre deux langues. La comparaison entre différentes situations de communication, par exemple entre des écrits composés pour soi et les siens et d'autres adressés à des interlocuteurs variés, restitue un cadre déjà plus complet du maniement des langues. Les rares archives marchandes conservées dans l'aire ligure, piémontaise et lombarde pour les XIV ${ }^{\mathrm{e}}-\mathrm{XV}^{\mathrm{e}}$ siècles, ${ }^{4}$ comme les échanges épistolaires entre des négociants de cette zone, révèlent ainsi que l'usage local consiste à rédiger tous les documents privés en latin, alors que les marchands d'autres régions italiennes utilisent depuis longtemps les scriptae vernaculaires. Si la langue que les premiers pratiquent est aussi appelée latinum/-o ou grammatica, ${ }^{5}$ elle se carac-

1 Petrucci, Le biblioteche antiche; Bec, Les livres des Florentins.

2 Par exemple Weissen, Ci scrive in tedescho!; Bottin, La pratique des langues; Guidi Bruscoli, I mercanti italiani.

3 Après les nombreux travaux d'A. Castellani et d'A. Stussi, voir parmi les études récentes Tomasin, Testi in italiano antico; Tomasin, Sul contatto linguistico nella Romània medievale; Brown, Early evidence for tuscanisation; Brown, Multilingual merchants.

4 Zerbi, Il mastro a partita doppia; Gioffrè, Lettere di Giovanni da Pontremoli.

5 Zanobi di Forese, facteur de l'agence Datini d'Avignon, explique que le marchand piémontais Michele di Nono souhaite que Francesco Datini lui écrive en latin: «La lettera mi desti venia qui a Michele di Nolo, a lui la die' e lessila chome me ne disse. E perché e' sanno ma. legiere nostre lettere, arebono charo scrivesi in gramaticha. Sì che per l'avenire di chosa portasse mandate la lettera in gramaticha» (ASPo, Archivio Datini [ci-dessous ASPo, D.] 425, 504441). Le marchand milanais Giovanni da Pessano écrit: «Son achostumato scrivere latino, sì che no[n] ve maraveliatti s'i' no[n] son bon scritore in romano, però che no• l'ò achostu- 
térise par une forte variation morphologique et une proximité syntaxique avec les vernaculaires qui la différencient du latin plus standardisé utilisé par les lettrés des mêmes régions. Avec ces formes vernaculaires du latin, il convient donc d'ajouter à l'opposition classique entre la langue parlée, sous ses diverses variantes, par tous et écrite par les marchands, et la langue écrite par les plus instruits une autre frontière diglossique qui traverse le latin et distingue la scripta des marchands de l'Italie du nord-ouest de l'écriture des notaires, clercs et lettrés.

Des notations ponctuelles, contenues surtout dans les correspondances, permettent de brosser le panorama linguistique au sein duquel agissent les marchands toscans dans leur métropole et sur les places de la Méditerranée occidentale. Vers la fin du XIV ${ }^{\mathrm{e}}$ siècle, ils ne disposent qu'exceptionnellement d'une maîtrise du latin dépassant la copie de quelques brèves formules, surtout religieuses. Francesco Datini avoue parfois son ignorance de cette langue, par exemple lorsqu'il ajoute sur la traduction vernaculaire par le notaire ser Martino di Giovanni Guiducci du testament de son associé Boninsegna di Matteo: «è in volghare perché non sapiamo gramaticha». ${ }^{6}$ Boninsegna expliquait lui-même lors d'une procédure juridique menée contre la Chambre apostolique d'Avignon avoir recours à un autre notaire, son cousin Lorenzo di Buto Tedaldi: «i' ò menatto mecho ser Lorenzo di Buto perché intende la gramaticha ed io noe». ${ }^{7}$

A l'occasion des recrutements des facteurs des agences, c'est de façon exceptionnelle qu'on évoque la connaissance du latin chez un candidat, comme Rosso d'Andrea da Gambassi. L'associé qui le recommande souligne par ailleurs que cette connaissance est un atout supplémentaire, non un critère essentiel comme les compétences graphiques et comptables ou la loyauté et le dévouement. ${ }^{8}$ Si la maîtrise de vernaculaires étrangers peut aussi être évo-

mato» (ASPo, D.341, 59). Un marchand de la région de Parme écrit: «Et de hoc placeat michi scribere per vestras literas magis legibiles quam fuerunt iste quam mixistis, faciendo ipsas scribi literaliter pro maiori intelligentia si potestis. (...) Ideo precor quod in hoc de vestra intentione rescribere placeat per vestras literas literaliter descriptas, quia vulgariter eas male scio legere» (Greci, Notizie sul commercio parmense, p. 579).

6 ASPo, D.1169/4, 3. Francesco écrit à l'un de ses dépendants d'obtenir d'un médecin des précisions sur un régime médical proposé à sa femme en les notant lui-même: «e sscrivi ch[i]aro e noi lo 'ntenderemo melglo che noe faremo i- latino» (ASPo, D.695, 309504). On peut rapprocher de ces citations l'affirmation de Domenico Lenzi dit il Biadaiuolo: «latino mai mia lingua apprese» (Petrucci, Miglio, Alfabetizzazione e organizzazione scolastica, p. 477).

7 ASPo, D.321, 2587.

8 «Quando Franciescho diliberò Lodovico no[n] fossi, diliberò mandarti di qua in ischanbio di lui e per le scriture in tuo aiuto uno giovane abiamo co noi tenuto più tempo al chonto della cassa, il quale è bene carico delle scriture, apresso bonisimo scritore, altrimenti che Nicolò 
quée lors des recrutements, ${ }^{9}$ on rencontre beaucoup moins qu'à la période moderne des efforts conscients pour l'acquérir à travers un enseignement dans la ville d'origine, la circulation de manuels bilingues ou un séjour à l'étranger motivé par cette finalité. ${ }^{10}$

La situation linguistique varie par ailleurs selon les places. Florence est reliée par des contacts épistolaires réguliers aux grandes villes italiennes, européennes de l'ouest et méditerranéennes mais reste peu fréquentée par les négociants étrangers. Venise, Avignon, Barcelone ou Bruges entretiennent en revanche un milieu d'affaires cosmopolite composé à la fois de négociants locaux et italiens fixés sur place et de voyageurs en mission. D’autres itinérants professionnels, comme les hommes d'armes fréquentant la boutique Datini d'Avignon, viennent d'horizons encore plus variés. Pour insister sur la lourdeur de ses tâches, un associé la caractérise comme une tour de Babel: «poi ci viene $\mathrm{i}[1]$ dì $\mathrm{C}$ presone [= persone], e voglono di C chose, e l'uno parla bertone [= bretone] e l'atro berghamascho». ${ }^{11}$ Une opposition ultérieure apparaît entre les langues romanes, d'acquisition plus aisée pour des Italiens, et les parlers germaniques, slaves ou arabes, auxquels certains restent imperméables même après un séjour prolongé et qui imposent des truchements plus systématiques. Entre langues romanes, la pratique du vernaculaire local paraît à peu systématique à l'oral et, de manière au moins passive, à l'écrit. Mais elle affecte différemment l'expression écrite. A part l'évocation de quelques proverbes appris sur place, ${ }^{12}$ les écrits de Francesco Datini ne révèlent pas vraiment l'in-

\footnotetext{
Vespuci, apresso bonisimo ragioniere; e oltra ciò sa latino, che anche viene a punto molte volte. Ma quello di che più siamo contenti si è che noi l'abiamo trovato leale e di bonisima condizione, chon amore alle cose della compagnia, apresso reverente e ubidente a' suoi magiori quando [= quanto] si dee » (ASPo, D.984, 703355; Melis, Aspetti, pp. 113-114).

9 Notamment à propos du jeune Florentin Tuccio di Gennaio, déjà familiarisé à la langue et à la société catalanes, que le dirigeant du comptoir de Barcelone veut faire recruter par Francesco Datini: «E perché costui è uxo di qua e intende bene la lingua e à 'sai praticheza pertutto e per molti caxi posono adivenire, non potrei avere miglore compagnia; ché faciendo ora venire uno fanciulo di costà o da Pixa, starebe 6 mesi anzi intendese il parlare o lla conoscienza» (ASPo, D.635, 10173; Melis, Aspetti, p. 256). Ou à propos d'Agnolo di Iacopo: «Il gharzone che Lucha tolse a Valenza più tenpo fa è qui in chasa; e promettovi che, se ll'aveste a chostumare, e' vi piacierebbe; puosi fare di lui chome d'un fanc[i]ullo di 12 anni e sa chosì i paesi de- reame chome l'andare per chosa [= chasa]; e à la lingua, che è un piaciere» (ASPo, D.666, 508670; Melis, Aspetti, p. 260).

10 Weissen, Ci scrive in tedescho!; Hoock, Kaiser, Les manuels plurilingues; Bottin, La pratique des langues.

11 ASPo, D.427, 407267.

12 «Diciesi a Vingnone che ne' fatti della guerra, cholle bugie ti ghoverna» (ASPo, D.699, 410086); «e sai che a Vingnione dicie uno proverbio che allo infornare si fa il pane chornuto» (ASPo, D.647, 107985).
} 
fluence de son séjour provençal de 30 ans. ${ }^{13}$ Mais certains Toscans d'origine nés ou éduqués en terre provençale ou catalane ne savent plus maintenir la frontière entre le toscan et la langue locale. A Avignon Niccolò di Francesco Pentolini emploie par exemple «yeu» pour «io» ou «car» en lieu de «però». A Perpignan Piero Tecchini écrit dans un catalan hybridé de quelques italianismes plus que l'inverse. ${ }^{14}$ Sur ces places, avec l'emploi alternatif des deux langues ou leur confusion, on observe aussi leur usage concurrent au sein d'un groupe pour des textes standardisés. Maîtres et trésoriers de la confrérie avignonnaise Notre-Dame-la-Majour tiennent ainsi selon leur origine en toscan ou en provençal les comptes de l'institution, dont le lexique se limite à quelques types d'entrée (cotisations, amendes, funérailles, legs, fourniture de cire, etc.). ${ }^{15}$ A une plus large échelle, c'est le cas de la lettre de change, bref écrit adressé dont les variantes se limitent pratiquement à l'identité des parties et des lieux, aux montants et unités monétaires, au délai de paiement et à la date. ${ }^{16}$

\section{L’Archivio Datini comme terrain linguistique}

Pour restituer une image plus complète des papiers divers produits par les marchands ou circulant entre leurs mains, il conviendrait cependant d'atteindre la «maison des écritures», ${ }^{17}$ c'est-à-dire l'ensemble des écrits assumant des fonctions diversifiées qui emplissaient à la fois leurs comptoirs et leur domicile. Une cartographie des divers types d'écrits permettrait alors de vérifier quelles typologies étaient plus investies par le latin, quels usages assuraient de tels documents, à quels truchements avaient recours des marchands qui maîtrisaient difficilement cette langue, pour obtenir ces écrits comme pour les interpréter. L’Archivio Datini présente l'un des meilleurs terrains disponibles pour une telle enquête, dans la mesure où ses séries, léguées directement par leur détenteur à la fondation qui lui succédait dans son palais de Prato, ont d'autant moins été dispersées qu'elles

13 Sauf peut-être le provençalisme «tesiari» < tesseire, tesseyre, 'tisserand': «s'io non guardase ongni ischartabello e chonti di Niccholò dell'Amanato e forse di pue altri, chome sono tesiari e altra giente» (ASPo, D.675, 9440).

14 Sur Piero di Matteo Tecchini, voir dans l'attente d'une véritable étude une caractérisation de ses missives par Tomasin, Testi in italiano antico, p. 403-404.

15 Archives départementales de Vaucluse [désormais ADV], Archives hospitalières d'Avignon, Majour, E4.

16 De Roover, L'évolution de la lettre de change; Bottin, La pratique des langues, pp. 84-85.

17 Pour reprendre la formulation de l'ouvrage Ecritures ordinaires, p. 31. 
sont pour la plupart restées oubliées du milieu du XVI ${ }^{\mathrm{e}}$ siècle à $1870 .^{18}$ Autour des papiers de Francesco Datini (1335 ca.-1410), marchand d'origine obscure, enrichi d'abord à Avignon, puis à partir de 1383 comme dirigeant d'un réseau d'agences implantées dans la Méditerranée nord-occidentale ont été réunies les archives des agences périphériques. Très concentré du point de vue chronologique, le fonds documente surtout les trois dernières décennies de la vie de Francesco.

En dépit de quelques pertes comme les manuscrits littéraires et les correspondances jadis amassées à Avignon, l'éventail typologique préservé est l'un des plus larges observable dans des archives d'origine privée, au point de s'étendre à des feuillets éphémères comme des listes de tâches à accomplir. Une autre caractéristique est l'extrême diversité des scripteurs, non seulement du point de vue linguistique (en dehors du latin, principalement le toscan mais également quelques autres dialectes italiens, le catalan, un peu le provençal, et de façon sporadique le français, le flamand, le castillan et l'arabe) mais aussi des origines géographiques et des conditions sociales: à côté d'une majorité de marchands et prestataires de services, apparaissent des artisans, rarement des cultivateurs, mais divers notaires, lettrés ou religieux, quelques prélats et seigneurs, un certain nombre de femmes et parfois des enfants.

Le fonds Datini se compose principalement d'environ 600 registres ou liasses de cahiers de comptes et d'autant de liasses contenant surtout les séries épistolaires (environ 160000 lettres) avec des documents techniques ou juridiques. Pour le repérage des documents latins, en nous appuyant sur l'inventaire disponible sur le Datini on line, nous nous efforcerons d'atteindre aussi les dernières liasses du fonds, peu consultées et dont beaucoup ne font pas encore l'objet d'un inventaire détaillé.

On peut catégoriser sommairement les écrits produits par les marchands ou circulant entre leurs mains à partir de leur fonction première. Elle n'empêche naturellement pas la reconversion de certains à d'autres usages. La correspondance reçue par Francesco atteste notamment à travers les notes dorsales qu'il apposait sur certains secteurs qu'elle pouvait être relue des années après l'expédition, reclassée différemment, indexée, voire produite en justice. ${ }^{19}$ Instrument de la communication, la correspondance archivée devient alors le support d'une mémoire polyphonique, parfois un écrit probatoire. Mais la fonction première pèse davantage dans le choix des scripteurs et des langues du document. On peut donc résumer la correspondance entre la typologie de ces écrits, le milieu de leur production et leur langue au moyen du tableau suivant.

18 Hayez, L'Archivio Datini.

19 Hayez, L'Archivio Datini, p. 186-189. 
Tableau 1: Les écrits conservés dans les demeures et agences des marchands.

\begin{tabular}{|c|c|c|c|}
\hline fonction & typologie & producteurs & langue \\
\hline gestion & $\begin{array}{l}\text { livres, cahiers et feuillets de } \\
\text { comptes } \\
\text { listes de planification et de contrôle }\end{array}$ & $\begin{array}{l}\text { marchands, } \\
\text { artisans, } \\
\text { autres }\end{array}$ & $\begin{array}{l}\text { vernaculaire } \\
\text { latin vernac. (Ligurie, } \\
\text { Piémont, Lombardie) }\end{array}$ \\
\hline communication & $\begin{array}{l}\text { lettres personnelles et de } \\
\text { compagnie } \\
\text { pièces-jointes (prix-courants, } \\
\text { cargaisons, . . .) } \\
\text { corresp. spécialisée (lettres de } \\
\text { change, de voiture, ordres de } \\
\text { paiement, . . .) }\end{array}$ & $\begin{array}{l}\text { marchands, } \\
\text { aussi autres } \\
\text { pour lettres } \\
\text { personnelles }\end{array}$ & $\begin{array}{l}\text { vernaculaire } \\
\text { latin vernac. } \\
\text { (marchands ligures, } \\
\text { piémontais, lombards) } \\
\text { latin (notaires, clercs) }\end{array}$ \\
\hline thésaurisation & $\begin{array}{l}\text { textes religieux et littéraires, } \\
\text { formulaires } \\
\text { savoirs pratiques (recettes, } \\
\text { calendriers, manuels commerciaux, } \\
\text { itinéraires, . . .) }\end{array}$ & $\begin{array}{l}\text { lettrés } \\
\text { marchands et } \\
\text { lettrés }\end{array}$ & $\begin{array}{l}\text { traduction vernaculaire } \\
\text { (parfois latin) } \\
\text { vernaculaire (et latin } \\
\text { pour recettes) }\end{array}$ \\
\hline probation & $\begin{array}{l}\text { correspondance d'institutions, } \\
\text { lettres patentes, bulles, laissez- } \\
\text { passer } \\
\text { consilia sapientis } \\
\text { procédures et sentences }\end{array}$ & $\begin{array}{l}\text { marchands, } \\
\text { artisans, lettrés } \\
\text { notaires }\end{array}$ & $\begin{array}{l}\text { vernaculaire (plus } \\
\text { rarement latin) } \\
\text { latin (traduction } \\
\text { vernaculaire pour } \\
\text { résumés et brouillons) } \\
\text { latin, parfois } \\
\text { vernaculaire } \\
\text { latin } \\
\text { latin, parfois vernaculai }\end{array}$ \\
\hline
\end{tabular}

\section{Les écrits de gestion}

Les écritures de gestion se composent principalement des comptabilités et autres écrits d'enregistrement, tenus sous diverses formes matérielles (livres, cahiers, feuillets volants, relevé de compte intégré dans la forme épistolaire). Très variés du point de vue de leur technicité, ceux du fonds Datini sont néanmoins presque exclusivement tenus par des marchands et boutiquiers, rarement par des artisans ou des lettrés. La grande majorité des scripteurs sont des Toscans, même si l'on peut trouver sporadiquement quelques entrées de mains étrangères, comme celles du facteur castillan Juan de Burgos à Avignon ${ }^{20}$ ou des registres secondaires

20 ASPo, D.63, fol. 39, 41v, 42-v, 44v. 
plus ouverts aux partenaires de transactions dans les agences de Majorque et Valence et la teinturerie de Prato. Des marchands génois présents à Majorque ou Valence peuvent alors y insérer quelques entrées latines, à côté d'interventions en catalan, hébreu ou arabe. ${ }^{21}$ Mais c'est surtout sous forme épistolaire que l'on rencontre quelques comptes latins, comme celui envoyé par Michele di Nono, marchand d'Avigliana, à l'agence d'Avignon. ${ }^{22}$ On voit donc différents filtres renforcer l'utilisation presque exclusive du toscan dans les écrits d'enregistrement: le personnel des agences est presque entièrement recruté dans cette région; les comptabilités sont tenues per ce personnel selon des modèles définis par les dirigeants. Les partenaires extérieurs font souvent l'effort de passer à l'écriture vernaculaire, dès qu'ils travaillent régulièrement avec des Toscans.

Les écrits de planification et de contrôle assument souvent des formes spontanées qui recourent surtout à la typologie de la liste ou du tableau à deux colonnes. Ceux transmis par Francesco et ses dépendants sont inévitablement en toscan. Dans la catégorie des inventaires mobiliers, on note cependant des degrés divers de formalisation, à travers des formats différents (petit carnet, cahier de format courant mais dépourvu de reliure, feuillet simple ou double, etc.). ${ }^{23}$ Seul un des inventaires mobiliers conservés comporte, parmi les multiples entrées en vernaculaire, quelques-unes en latin, mais il s'agit d'un brouillon rédigé par le notaire ser Lapo Mazzei après la mort du marchand ${ }^{24}$ et son confrère ser Tieri da Larciano transpose plus tard ces entrées en toscan dans le registre de parchemin destiné à être exposé dans la fondation. ${ }^{25}$

\section{Les écrits de communication}

La correspondance du fonds Datini est constituée majoritairement de missives reçues et archivées par Francesco, ses agences et certains de ses dépendants et, pour une très faible part, de brouillons ou copies de lettres expédiées. Du point de vue linguistique, il n'y a pas de distinction entre les lettres privées, les textes adressés plus techniques (lettres de change, ordres de paiement, lettres de voiture,...) et les pièces-jointes telles les prix-courants ou les cargaisons de navires, hormis la plus

21 Pour l'agence de Valence en 1400, voir ASPo, D.954 ; multilinguisme décrit pour l'agence de Majorque par Houssaye, Langue écrite et langue orale.

22 ASPo, D.181, 317173.

23 Palazzo Datini a Prato, 2, p. 581-598.

24 ASPo, D.1177/3, fol. 1-5.

25 ASPo, Ceppi, 1618, fol. 35-55v; Palazzo Datini a Prato, 2, pp. 598-612. 
grande richesse lexicale et stylistique des premières, ouvertes à des contenus plus divers. Les correspondances constituent un secteur plus ouvert au latin, du fait de la très grande variété d'origine des centaines d'expéditeurs, ainsi que de la diversité de leur condition sociale et de leur formation culturelle. Toutefois les lettres latines de tout type ne représentent que 907 unités, sur environ 160 000. En répartissant ces missives latines par localités de provenance apparaît déjà l'importance des régions du Piémont et de la Ligurie dans cette série (Fig. 8.1).

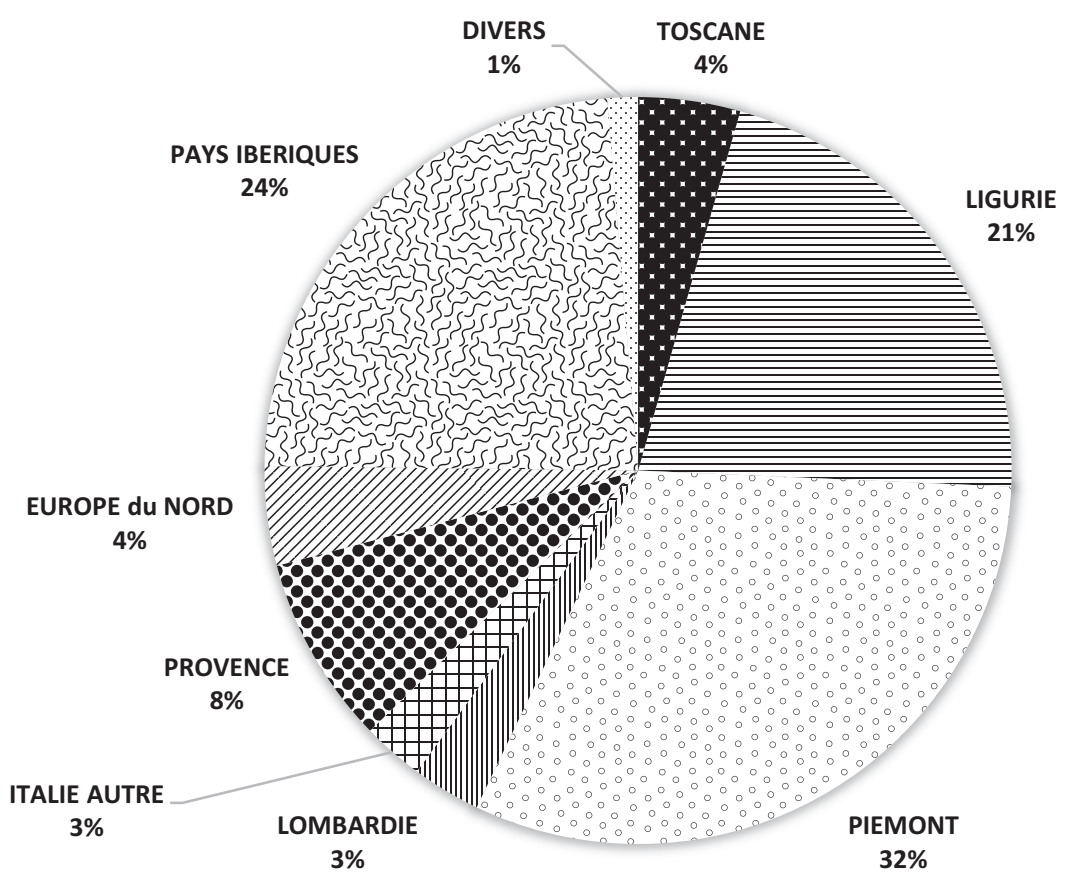

Figure 8.1: Localités d'origine des lettres latines du fonds Datini.

Dans certaines aires, ibérique ou flamande notamment, les correspondants du réseau toscan sont plus souvent italiens que locaux. Si l'on pousse plus loin la classification en se risquant à attribuer une origine régionale aux expéditeurs des lettres latines selon divers critères onomastiques, la concentration est de fait beaucoup plus resserrée sur les marchands ligures et piémontais (Fig. 8.2). En résidence durable à Gênes, les facteurs de l'agence Datini apprennent inversement à se débrouiller pour écrire des missives ou des comptes en latin vernaculaire. Quelques lettres latines ont ainsi été laissées par Andrea di Bonanno et Luca del Sera, Florentins, et Ambrogio di 


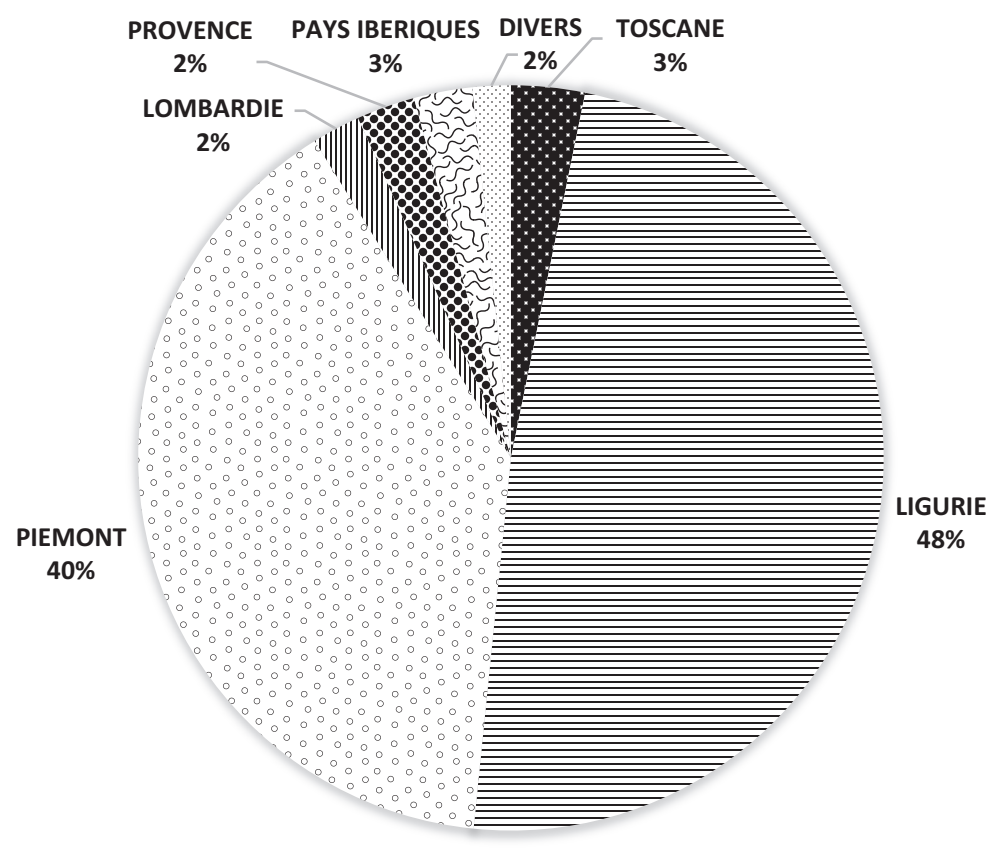

Figure 8.2: Origine des expéditeurs des lettres latines du fonds Datini.

messer Lorenzo de' Rocchi, Siennois. ${ }^{26}$ Quelques Ligures, comme le changeur génois d'Avignon Federigo Imperiale, apprennent à écrire aussi en vernaculaire et peuvent opérer la commutation linguistique selon leur interlocuteur. ${ }^{27}$ Ceux qui écrivent en latin à des Toscans sont en revanche surtout des correspondants occasionnels, qui se trouvent aussi bien dans l'aire catalane, en Flandre ou sur le littoral tyrrhénien, et adressent souvent des textes brefs et standardisés comme des lettres de change. Les Piémontais sont également des correspondants occasionnels du réseau, plus dispersés entre diverses villes de leur aire d'origine, la Ligurie, le littoral toscan, la Provence

26 ASPo, D.1116, 6000680 et ibid., 127778 et 127777 pour le premier, à qui sont aussi adressées beaucoup des lettres latines expédiées par des marchands lombards et piémontais; D.1116, 424660 pour le deuxième; Dini, Una pratica di mercatura in formazione, p. 5 et pl. I pour le troisième; sur la résidence de celui-ci à Gênes dans les années 1387-1392, ibid.., p. 6.

27 Ont été conservées de lui deux lettres latines à un autre Génois (ASPo, D.429, 500107 et 9293317) et trois lettres vernaculaires aux compagnies Datini de Gênes et de Majorque (D.746, 416599; D.1045, 801037-801038). 
et le Languedoc. Un cas original est présenté par «Odun de Cavernay», libraire-copiste d'origine piémontaise qui choisit le français pour s'adresser occasionnellement aux Toscans de l'agence Datini de Barcelone. ${ }^{28}$ Le formulaire de l'unique missive conservée de sa main suggère comme le choix de la langue qu'il était davantage inséré dans un milieu érudit parisien qu'au sein des réseaux marchands italiens et avait peut-être perdu l'habitude d'utiliser le latin dans les écrits privés qu'il devait tenir, parallèlement à la copie de manuscrits littéraires français. Le faible nombre de lettres latines provenant de marchands lombards s'explique en revanche par le fait que les correspondants du réseau Datini dans des villes de cette région comme Milan, Côme, Bergame, Brescia, Plaisance ou Parme, quoique fréquemment lombards et de ce fait accoutumés à rédiger leurs écrits en latin, se mettent au vernaculaire dès qu'ils coopèrent régulièrement avec des collègues toscans. ${ }^{29}$

Un second groupe de correspondants latinistes est formé par les notaires, surtout toscans, les religieux d'origine variée et les seigneurs de la France méridionale fréquentés par Francesco Datini à la cour pontificale, qui entretiennent encore avec lui des rapports épisodiques. Notaires et religieux toscans écrivent toujours au marchand en vernaculaire, mais il peuvent conserver par habitude ou souci de distinction quelques formules latines, entre la salutatio, la formule de congé, la souscription ou l'adresse extérieure. ${ }^{30} \mathrm{De}$ façon plus exceptionnelle chez les correspondants de Francesco Datini, le gardien du couvent franciscain de San Miniato, Francesco da Poppi, insère quelques passages scripturaires latins dans une lettre d'édification. ${ }^{31}$ Significatif est le cas d'un religieux pisan qui, pour correspondre en latin avec le prieur de San Basilio degli Armeni, doit néanmoins inscrire en vernaculaire sur la face extérieure de la missive à l'intention du messager chargé de la porter l'adresse de ce couvent florentin moins connu que d'autres: «honesto religioso fratri Johanni priori conventus Sancti Basilii Armenorum Florentie, al canto alla Macina, detur». Deux religieux seulement adressent à Francesco Datini une missive latine. L'un, son ancien confesseur Jean Sicard, est un frère mineur et théologien provençal. ${ }^{32}$ L'autre est écrite par un «frater Johannes»

28 Arnoux, Bourlet, Hayez, Les lettres parisiennes, pp. 220-221.

29 Brown, Early Evidence for Tuscanisation. Voir également les citations contenues dans la note 5 .

30 Pour les notaires, voir Ser Lapo Mazzei, Lettere di un notaro et Brambilla, Hayez, La maison des fantômes; pour les religieux, Brambilla, 'Padre mio dolce'.

31 ASPo, D.1092, 6000551; Brambilla, 'Padre mio dolce', p. 138.

32 ASPo, D.1102, 132286. Il faut l'identifier avec le «frate Giovanni de' frati minori che confesa Franciescho» de la fin du séjour avignonnais du marchand (Hayez, L'Archivio Datini, p. 129). 
d'origine inconnue, représentant le tiers ordre franciscain à Calvi pour réunir la rançon d'un groupe de captifs des Maures. ${ }^{33}$ Ils peuvent donc l'un comme l'autre choisir cette langue à défaut d'un vernaculaire commun bien maîtrisé.

Notaires, juristes, religieux et autres lettrés s'écrivent en revanche volontiers en latin entre eux, pour marquer leur appartenance à une classe valorisée. ${ }^{34}$ Dans le cas des nobles, les enjeux ne sont plus seulement de se faire comprendre, mais aussi de repérer qui dans leur entourage, clerc ou chancelier selon leur position et leurs ressources, va prendre la plume ou interpréter une missive reçue dans un vernaculaire étranger. Les rapports épistolaires entre Francesco Datini et le prince Louis II d'Anjou semblent par exemple avoir été largement relayés par une clientèle de clercs et lettrés qui comprenait suffisamment d'Italiens susceptibles d'écrire en cette langue. ${ }^{35}$ Francesco avait connu à Avignon dans l'entourage du lignage limousin d'Aigrefeuille le noble auvergnat Gui de Pesteil, qui menaçait à partir des années 1380 de le poursuivre en justice comme garant d'un autre marchand toscan. Avec ce seigneur de Branzac et Salers, les rapports étaient moins aisés, quand ils ne se limitaient pas à de simples messages oraux échangés avec les agents Datini. Quatre lettres écrites au nom de Gui ont été conservées dans le fonds, la première en latin mais avec une souscription provençale, les trois autres, tracées d'une autre main, entièrement en latin. ${ }^{36}$ Les brouillons de celles adressées par Francesco à ce chevalier sont tous en toscan: deux sont autographes, un d'une main non identifiée, deux autres sont des schémas de réponses proposés par ser Lapo Mazzei, notaire conseiller de Francesco, et par un marchand florentin retiré à Avignon, Nastagio di ser Tommaso. ${ }^{37}$ Les commentaires échangés entre Francesco et ses agents avignonnais expliquent que des lettres pouvaient ainsi circuler entre les deux correspondants, en latin ou en vernaculaire (toscan ou provençal selon le lieu de provenance), mais que leurs proches jouaient ensuite le rôle de truchement,

33 ASPo, D.1093, 1402737; Piattoli, Un tragico pellegrinaggio, pp. 269-270; Brambilla, 'Padre mio dolce', pp. 163-164.

34 Comme la missive de fra Matteo da Poppi à fra Francesco da Prato (ASPo, D.1114/2, 1403014; Brambilla, 'Padre mio dolce', pp. 241-242); on le note en particulier dans le noyau de correspondance passive du notaire de Prato ser Schiatta di ser Michele qui a été intégré aux archives de Francesco Datini (par ex. D.1114/1, 134733, 134992, 6000547, 1402830, 133057; et pour une autre lettre entre notaires de Prato, D.1114/2, 1402747).

35 Ainsi «messer Antonio di Villanova» (ASPo, D.1090, 127761), le Napolitain Buffilo Brancaccio (D.1091, 6100136 et 6100135), le juriste lucquois Giovanni Genovardi (D.340, 144) et «Rinaldo e Giacomo di messer Marco» (D.346/1, 1769).

36 ASPo, D.1095, 1402553, 509809, 509808 et 501089.

37 ASPo, D.1115, 9142612-9142614; ibid., 133133; D.1096, 9293286. 
pour le latin dans les deux cas, ou pour le toscan que les agents Datini traduisaient oralement au seigneur occitan. Le principal collaborateur de Francesco à Florence, Stoldo di Lorenzo, pouvait également intervenir entre Francesco et les agents avignonais pour superviser cette relation à risque.

Abiamo auto la lettera da questi di meser G(uido) che lasciò vi fosse mandata, e òlla vista (...). Voi vi sapete tutto e vedrete el tenore della lettera sua. Fate risposta per la prima. E perch'è in latino, vi bisongnerà ser Lapo. E per lo simile gli rispondete in latino, e fia buona lettera sì che la legha. ${ }^{38}$

Tu, Stoldo, di' a Boninsengna quelo che tti pare intorno a' fatti di Nicholò Pentolini e di messer Ghisi, e ricordagli che puote fare scrivere in latino e in volghare per mia parte quello che lgli pare si debia dire per me; e che di quanto farà sarò contento, chome s'io l'avessi scritto di mia propia mano. ${ }^{39}$

Dans ces textes vecteurs de la communication marchande, apparaît ainsi toute une casuistique. Les marchands toscans apprennent moins couramment vers 1400 des langues étrangères qu'ils ne le feront au XVI ${ }^{\mathrm{e}}$ siècle. Cependant les cas ne sont pas isolés où un marchand acquiert à la faveur d'un séjour durable à l'étranger la capacité non seulement de comprendre passivement la langue locale à l'oral et à l'écrit mais parfois aussi de la pratiquer dans la communication écrite, dès qu'il tisse des contacts fréquents avec le milieu commercial local. Dans le fonds Datini, on l'observe notamment pour le catalan (plus que pour le provençal ou le français, du fait de la perte d'une partie des archives de l'agence d'Avignon) ainsi que pour le latin vernaculaire chez les Toscans résidant à Gênes. Inversement quelques patrons de nef ou petits entrepreneurs provençaux ou catalans peuvent adopter une forme d'italien ou de langue hybride, parce qu'ils travaillent au sein d'un milieu majoritairement italien ou qu'ils se rendent eux-mêmes dans la Péninsule. Chez les marchands éduqués à l'usage exclusif du latin vernaculaire, c'est la fréquence et la régularité de leurs échanges avec des collègues vénitiens ou toscans qui détermine leur capacité à passer à une écriture italienne. L'intercompréhension plus large entre idiomes romans fonctionne assez couramment entre toscan et provençal et catalan (voire aussi le français), ainsi qu'avec le latin vernaculaire, mais surtout pour des textes brefs et standardisés. Enfin, un latin plus typique des lettrés et des praticiens de l'écrit administratif et judiciaire fait facilement fonction de langue de communication entre étrangers mais elle implique pour les marchands toscans l'assistance d'un notaire.

38 ASPo, D.630, 9291727.

39 ASPo, D.695, 309504. 


\section{Les écrits thésaurisés}

Parmi les savoirs recherchés et amoncelés prenait place la «bibliothèque» de Francesco Datini, en réalité un ensemble mouvant de textes religieux et littéraires tracés sur des supports variés, du codex au bout de papier, et par ailleurs réparti entre ses résidences de Prato et de Florence. Les manuscrits dotés de valeur commerciale ont été dispersés par la fondation peu après la mort du marchand; quelques feuillets volants sont en revanche demeurés dans le fonds. Parmi les textes mentionnés dans les inventaires du mobilier et surtout dans la correspondance échangée avec des proches, nous ne pouvons pas toujours déterminer ceux qui appartenaient en propre au marchand et ceux qui lui étaient prêtés par des proches. Et quelques vagues mentions, faisant par exemple allusion aux histoires des «antichi romani», peuvent se rapporter à des circulations orales autant qu'écrites..$^{40}$ Mais comme le précise un passage éloquent sur les velléités de Francesco de passer plus de temps sur ces manuscrits littéraires, il cherchait effectivement dans son âge mûr à amasser des recueils de littérature vernaculaire, souvent traduite du latin.

i' òe animo d'achostarmi a Dio melglo non ò fatto per lo pasato in pue modi; e per detta chagione chonpero molti libri in volghare per legiergli quando mi rincrescierà i fatti della merchatantia e per fare quello debo inverso Idio. Sono tutti libri che parlano di chose vertudiose, cioè sono tutti vangeli e pistole, il detto e lla vita di tutti santi e molte altre belle chose e buone; apreso quello disono molti valenti filosofi e altri valenti uomeni che lodarono le vertù e biasimarono vizi chome è Salamone, Aristotile, Patone [sic], Vergilio e Tuto Livo [sic] e Boezio e molti altri valenti uomeni romani e d'ongni nazione. ${ }^{41}$

On y dénombrait les Evangiles, un psautier, un livre d'heures destiné à sa femme, des lettres d'apôtres, des vies de saints, des Epîtres de Sénèque, un ouvrage dérivé de la Consolation de la philosophie de Boèce, l'oraison de Bède ainsi que les laudes de Iacopone da Todi, l'épître de Bernard de Clairvaux, la

40 «Richordoti chon fede che tue facci chome facevano i buoni romani che, tornati dal chonquisto in sue charri trionfali, non si verghognavano di tornare a llavorare la terra» (ASPo, D.1115, 9300474); «se ci fosse il podere e 'l sapere chom'è il buono animo, io ne 'nposcierei quelli antichi romani che moriano a diletto per lo loro chomune e fec[i]ono tante nobili chose ed erano infedeli; e noi che siamo cristiani ed abiamo tante legii e tanti vangieli, siamo la pue chativa gente che mai fosse» (D.182, 9142338); «Richordoti che i buoni antichi romani, quando erano presentati, rimandavano il presente indietro e dicevano voleano singnoregiare choloro di chi era il presente. E chome ch'io non sia di quelli buoni romani, pure s'io fossi stato a Prato, arei rimandato il presente e detto all'aportattore: "Io voglio vedere in persona chi manda il presente, se vuole ch'io l'acetti”» (D.1115, 9300406; Melis, Aspetti, p. 83).

41 ASPo, D.1086, 9142555; Melis, Aspetti, pp. 92-93. 
lettre de Giorgio camaldule, une prière de Brigitte de Suède, des lettres de Giovanni dalle Celle, la formule de confession de Luigi Marsili. Parmi les poètes, rhéteurs et chroniqueurs médiévaux, Pétrarque pour quelques sonnets, Coluccio Salutati pour une traduction de la lettre à Iacopo d'Appiano, Antonio Beccari, Antonio Pucci, Tommasuccio da Foligno, Scoppa de' Bostichi, Giovanni Quirini et quelques anonymes dont les poèmes sur la mort de John Hawkwood et la guerre des Huit Saints, ainsi que la chronique de Matteo Villani. ${ }^{42}$ Parmi les feuillets conservés, écrits dans des graphies et selon des mises en page très disparates, seuls un psaume et une grande partie de trois feuillets présentant plusieurs colonnes d'expressions proverbiales attribuées à divers auteurs antiques et médiévaux sont en latin. ${ }^{43}$

Un autre massif est constitué, parmi les écrits thésaurisés, de savoirs pratiques accumulés en des circonstances variées de la vie professionnelle et personnelle. Rédigés sur des cahiers ou des feuillets plus souvent que sous forme de registres, ils proviennent en grande partie, mais pas exclusivement, du milieu du négoce. Il semble que toutes les recettes médicales évoquées dans la correspondance n'aient pas été conservées. Il faut donc supposer que certains feuillets finissaient déjà du vivant de Francesco aux mains de ses praticiens.

Les manuels de commerce ont ici été conservés au stade embryonnaire des données amassées et classées dans les agences, plus que sous la forme de manuscrits soignés de bibliothèque sous laquelle on les connaît plus souvent. Diverses agences Datini nous ont ainsi transmis des éléments de ce type, provenant de mains marchandes mais le plus souvent extérieures aux agences Datini, entre le cahier assez complet mais archaïque qui aurait été conservé par l'agence de Pise, le petit manuel limité à la description de Majorque que composait Ambrogio de' Rocchi et divers feuillets dont l'un est explicitement copié par un Florentin de Marseille. ${ }^{44}$ Tout ce matériau, comme les passages du même type contenus dans les correspondances, est en vernaculaire car produit par des Toscans. Il en va de même pour les quelques feuillets issus d'un autre filon de l'éducation marchande, associant des règles et problèmes de mathématique appliquée aux questions commerciales, des tables de calcul, des calendriers. ${ }^{45}$

42 Piattoli, In una casa borghese; Piattoli, L'origine dei fondaci datiniani; Brambilla, Itinerari nella Firenze di fine Trecento; Brambilla, Nove sonetti; Brambilla, Libri e letture; Camescasca, Testi letterari.

43 Sur ces feuillets, ASPo, D.1174/14, 15248, 15249 et 152410; Camesasca, Testi letterari, pp. 111-128.

44 Ciano, La 'pratica di mercatura datiniana'; Dini, Una pratica di mercatura in formazione; ASPo, D.1174/2, 1284; D.1174/10; D.1173, 1205.

45 ASPo, D.1174/14, 1524 et 1527. 
Les recettes présentent en revanche un panorama linguistique plus varié car elles relèvent d'une pluralité de secteurs d'activité et impliquent des acteurs divers. Des recettes pratiques concernant les métaux précieux et des produits comme les draps de laine, les teintures et les pigments sont habituellement rédigées en langue vernaculaire. Mais c'est souvent le cas également de recettes médicales, qu'elles soient communiquées par un médecin à son patient ou par le truchement d'un marchand à une relation. Dans le fonds Datini, on en dénombre une douzaine, sur un carnet de tâches à accomplir mais surtout sur des feuillets volants, parfois encore insérés dans des lettres d'un médecin comme Lorenzo d'Agnolo Sassoli mais plus souvent de marchands. ${ }^{46}$ Un feuillet comportant diverses recettes en présente aussi une en traduction latine; ${ }^{47}$ une autre recette n'est envoyée d'Avignon à Barcelone qu'en latin par le marchand Nofri di Bonaccorso di Tano, qui l'a copiée de sa main. ${ }^{48}$ Ces dernières étaient visiblement destinées à être remises à un médecin pour consultation. On ne rencontre pas dans ces recettes, contrairement à certains recueils élaborés dans un contexte marchand ou aux brevi portés sur le corps, d'utilisation magique de formules latines plus ou moins estropiées et de nomina barbara pour renforcer l'efficace d'un rituel magique. ${ }^{49}$

\section{Les documents juridiques}

Plus que les fonctions d'enregistrement, de communication ou de collectionnisme, ce sont des pratiques administratives et juridiques qui ont suscité l'intégration de textes latins dans ces archives privées. Durant sa vie, le marchand de Prato fit rédiger plusieurs centaines d'actes notariés, reçut nombre de lettres et d'actes publics et se trouva impliqué dans des dizaines de procès devant des tribunaux provençaux, toscans, ligures ou catalans. Nous ne disposons pas encore d'une étude approfondie sur Francesco Datini comme personne juridique, mais ce ne sont pas les traces de ces aspects qui font défaut, entre son fonds d'archives et l'Archivio di Stato de Florence. Parmi la masse des écrits destinés à prouver un engagement, un avoir ou la possession d'un bien, confirmer un

46 ASPo, D.199, fol. 254; D.1166, 9302542 et 9302433; D.1174/13, 1502-1508; D.347, 3947; D.1111, 1403325; D.1102, 131873-131876; Ser Lapo Mazzei, Lettere di un notaro, 2, pp. 370-376; Latronico, Documenti medici.

47 ASPo, D.1174/13, 1508.

48 ASPo, D.1111, 1403325.

49 Il tesoro di un povero, pp. 260, 379-381, 384, 395, 399, 420-422. 
droit ou régler un conflit juridique, l'Archivio Datini nous a tout d'abord transmis l'une des plus belles collections d'actes sous seing privé (en toscan scritte ou polizze, en latin apodixie ou cedule), avec près de 600 pièces. ${ }^{50}$ Provenant à la fois du contexte domestique et patrimonial et de la pratique des affaires, elles contiennent des reconnaissances de dette et quittances ou des pactes et conventions diverses, mais surtout des assurances maritimes et sont majoritairement issues de Toscane et de Ligurie, plus marginalement d'autres régions (Provence, Catalogne). Comme les correspondances, la très grande majorité de ces actes privés est rédigée en vernaculaire, le plus souvent toscan. Parmi la quinzaine de contrats privés latins, on rencontre sporadiquement un artisan lombard et des marchands piémontais, ${ }^{51}$ mais surtout des étudiants, juristes et prélats étrangers, principalement provençaux et catalans. ${ }^{52}$ Quelques modèlestypes de contrats privés ont été spécialement rédigés par les notaires employés par le marchand de Prato, mais toujours en toscan. ${ }^{53}$

Même dans une ville comme Florence où l'écrit privé avait remplacé l'acte en forme publique pour un grand nombre de transactions, le recours au second restait nécessaire dans certaines circonstances. Parmi les très nombreux actes requis par Francesco Datini et ses agences, une grande part est restée confinée aux minutiers. Mais des expéditions (ou grosses) jadis conservées dans ses locaux ont été intégrées par sa fondation charitable aux titres de celle-ci pour finir aujourd'hui dans la série Ceppi di Prato du fonds Diplomatico de l'Archivio di Stato de Florence, tandis que cinq autres ont été oubliées parmi les papiers Datini. On distingue parmi ces expéditions environ 80 actes concernant directement Francesco et ses proches; d'autres titres y avaient été joints suite à l'acquisition d'un bien ou d'une succession, ou la gestion d'intérêts d'autres individus. Tous ces actes sont rédigés en latin, en Toscane comme en d'autres aires de la Méditerranée occidentale. L'accès des marchands à ces textes difficiles par leur langue, savante et juridique, et, lorsqu'ils étaient conservés à domicile, par leur matérialité (le rouleau de parchemin, conservé parmi des dizaines

50 Les assurances sont conservées dans les liasses ASPo, D.1158 et 1160; les autres contrats dans D.1170, mais on en rencontre d'autres dans D.1147 et de façon sporadique dans divers registres et liasses (D.179/14, D.1171).

51 ASPo, D.179/14 et Frangioni, Martino da Milano; D.1147, 9281393.

52 ASPo, D.1147, 9281391 et 9281255, 1067, 9303347, 9301173, 9300779, 9281392, 9281398, 9303348, 9281400, 9281405, 9281403, 9281404.

53 Voir principalement une formule d'obligation qui comporte également des gloses de plusieurs mains (ASPo, D.1170, 1211), mais aussi D.1173, 1231 pour la formule de souscription rédigée par ser Lapo Mazzei pour un tiers représentant de deux femmes analphabètes qui s'obligent envers Francesco Datini. 
d'autres, qu'il faut dérouler avant de voir son texte), était facilité par deux expédients. Le client notait souvent dans un livre de comptes ou de famille l'objet de la transaction, le nom du notaire et la date. ${ }^{54}$ Ce résumé servait notamment à retrouver le notaire, si l'acte n'avait pas été grossoyé antérieurement. ${ }^{55}$

Ricordanza chome lunedì mattina a terza a dì VIII d'aghosto 1373 io Franciescho di Marcho da Pratto mi conpromisi con Tuccio Lanbertucci, carta per mano di ser Andrea da Mellano, testimone Domenicho chalzettiere e uno suo gharzone. ${ }^{56}$

Pour identifier le parchemin recherché parmi les plus ou moins nombreuses expéditions détenues dans son coffre, un marchand se repérait en revanche grâce aux notes dorsales qu'il y avait inscrites ou fait inscrire. Le plus souvent tracées en vernaculaire, ces notes constituent une traduction de l'acte résumé à son objet. Dans des séries comme celles de Francesco et d'autres compatriotes marchands, leur typologie graphique est plus souvent la mercantesca que celle d'un notaire, et leur syntaxe se réfère parfois clairement au client concerné ou à ses proches.

Charta de la chasa grande chonperamo da Bartolomeo di Dolfo. ${ }^{57}$

Carta di quitanza di $\mathrm{V}^{\mathrm{C}}$ fior. facta a Francesco da Prato per Bernardo de’ Rossi. ${ }^{58}$

Dans les archives Datini, l'acte notarié n'était pas seulement présent à travers les mentions contenues dans les livres et la correspondance et par ces expéditions. Quelques fragments de brèves latines de notaires florentins et avignonnais y sont parvenues par des circulations plus ou moins difficiles à reconstituer. ${ }^{59}$ On y trouvaient aussi des copies non authentifiées ou attestations d'actes par les notaires, des traductions, des résumés et des ébauches ou formules d'actes qui attestent du travail d'ajustement aux divers contextes géographiques et socio-juridiques et de

54 Comme le recommandait aussi Paolo da Certaldo: «Sempre quando fai fare alcuna carta, abbi un tuo libro e scrivivi suso il dì che si fa e 'l notaio che la fa e 'testimoni, e 'l perché e con cui la fai, che la ritruovino. E a fuggire molti casi e pericoli de’ falsi uomini, sempre si vorrebbe fare compiere; e tiellati ne la cassa tua compiuta» (Paolo di Pace da Certaldo, Libro di buoni costumi, p. 46).

55 La procédure est illustrée a contrario par un exemple où le nom du notaire a été oublié sur le registre, et que les dépendants de Francesco Datini ne retrouvent qu'avec difficulté (Brambilla, Hayez, La maison des fantômes, pp. 107-108).

56 ASPo, D.57, fol. 112v.

57 ASFi, Diplomatico, R. Acquisto Strozziane Uguccioni, 18-3-1364.

58 ASFi, Diplomatico, Ceppi di Prato, 26-3-1379.

59 Dans le cas des fragments de brèves avignonnaises de ser Lorenzo di Buto Tedaldi de 1379-1380 (ASPo, D.1174/3, 1302), il est possible qu'elles se soient égarées dans l'agence Datini locale dirigée par son cousin Boninsegna di Matteo après l'expulsion de ce notaire invalide de sa maison par l'autorité apostolique vers 1387 (ASPo, D.322, 2706). 
la communication entre praticiens et clients pour produire l'acte, mais aussi le commenter et le poursuivre par d'autres actions. ${ }^{60}$

La correspondance administrative, produite par les chancelleries communales et princières, les organisations de métiers, consulats et juridictions, ou plus rarement adressées à elles, a laissé dans ces archives privées des vestiges plus importants qu'on aurait pu le supposer, avec environ 60 pièces. ${ }^{61}$ Ces lettres patentes ou adressées à un titulaire d'office ou un particulier, bulles, suppliques, privilèges, laissez-passer, etc. attestent des droits ou les octroient, recommandent Francesco Datini comme justiciable ou participent d'une procédure. La langue choisie relève ici de la pratique des diverses institutions. Si les lettres des chancelleries princières ou d'autorités provençales, françaises et catalanes sont le plus souvent en latin, des institutions toscanes comme la Seigneurie de Florence utilisent le vernaculaire (avec une adresse et une datation latines), quand elles s'adressent à leurs ressortissants, quelle que soit leur résidence, ou à des magistrats comme des podestats et des notaires occupant une charge dans l'Etat, mais le latin, quand elles écrivent à des juristes ou des autorités étrangères.

Un dernier secteur du fonds peut être reconstitué à partir de la documentation judiciaire. Les dizaines de procès dans lesquels Francesco Datini a été impliqué à un titre ou un autre dans diverses régions ne sont tous représentés ici. On en trouve d'autres dans des archives institutionnelles, comme les actes du tribunal de la Mercanzia de Florence; d'autres encore ne sont plus connus que par quelques mentions dans les comptes, correspondances ou actes notariés. Et certains documents judiciaires parvenus dans le fonds Datini n'impliquent que des connaissances du marchand dont il gérait des intérêts ou dont il finit par capter les papiers. Comme pour les documents notariés, ce secteur témoigne aussi du travail préparatoire effectué dans l'entourage du justiciable, avec l'exposé de cas juridiques apparemment présentés à des juristes, ${ }^{62}$ quelques consi-

60 Voir par exemple le brouillon rédigé par ser Lapo Mazzei de la dotation de la nièce de Francesco [Caterina di Niccolò dell'Ammannaro Tecchini] (ASPo, D.1173, 1229), deux attestations par ser Lapo Mazzei d'actes reçus par lui (ASPo, D.1169/4, fol. 14 et 1169/5, 14-9-1400) et comme traduction vernaculaire le testament de Boninsegna di Matteo, communiqué à Francesco sous cette forme avec des copies latines non authentiques du testament du neveu Priore et d'autres actes concernant la mère de ce dernier, pour préparer le procès qui opposait Francesco aux héritiers de ces anciens employés (voir ci-dessus, note 6).

61 On les rencontre principalement dans ASPo, D.1173 et D.1169, mais aussi sporadiquement dans d'autres liasses, comme D.1114/1, 134730, D.1114/2, 1401136, D.1101, 134967, etc.

62 Comme la succession de Lotto di Simone degli Agli sur laquelle son gendre Nastagio di ser Tommaso demande un avis (ASPo, D.1174/3, 1343). 
lia sapientis ${ }^{63}$ et de nombreuses gloses apposées par des notaires sur certaines procédures.

La langue employée pour les procédures, issues de juridictions aussi diverses que l'audience de la Chambre apostolique, le Petit-Scel de Montpellier, les podestats de Florence et de Prato, la cour des marchands et le Consulat de la mer de Pise, la Mercanzia et divers métiers (arti) de Florence, certaines juridictions de Barcelone, Majorque et Valence, est le plus souvent souvent le latin, avec des passages vernaculaires dans certaines procédures provençales et catalanes et, pour la Mercanzia, l'usage plus systématique du toscan depuis $1355 .{ }^{64}$ Alors que les procédures de ce dernier tribunal, couramment étayées par la production de papiers d'affaires en toscan, semblent bien rodées, il n'en va pas de même pour le plus gros dossier judiciaire transmis par l'Archivio Datini, un registre d'environ 300 feuillets qui retrace un différend né dans les années 1360 entre deux marchands toscans d'Avignon, Ghieri d'Andrea de Pistoia et Bonaccorso di Vanni de Prato, après la dissolution de leur compagnie. La procédure est menée par l'auditeur de la Chambre apostolique Bernard de Rodez et a fini entre les mains de Francesco Datini, à qui Bonaccorso avait confié ses intérêts en partant en 1377 à Rome, où il allait bientôt mourir. ${ }^{65}$ Dans le texte latin sont insérées plusieurs dizaines de documents très divers, allant des habituelles cédules produites par les procureurs des parties jusqu'à des écrits privés des marchands, des actes notariés avignonnais et florentins, une lettre de bourgeoisie authentifiée en français par le garde de la Prévôté de Paris, un sauf-conduit délivré par Antoine seigneur de Beaujeu et une autre lettre administrative française, Bonaccorso ayant passé quelques années dans la capitale du royaume de France. Parmi les écrits marchands figurent quelques comptes et une vingtaine de lettres échangées entre les associés. L'oscillation entre la copie directe des trois documents français, d'une lettre et d'un compte toscans et la traduction latine d'un autre compte et d'une vingtaine de missives des mêmes marchands apparaît déjà surprenante. ${ }^{66}$ Mais le caractère souvent incompréhensible des textes toscans, voire de leur traduction frappe encore davantage. Alors qu'Avignon comptait à cette époque plusieurs dizai-

63 Notamment ceux d'Angelo degli Ubaldi et de Francesco Ramponi concernant la double imposition de Francesco Datini par le fisc florentin, tant comme ressortissant du contado que comme citoyen contraint (ASPo, D.1169/3). Une copie informelle latine d'un autre consilium concerne la succession de deux frères épiciers en 1365 (ASPo, D.1174/14, 1529).

64 ASPo, D.1168, 1169 et 1173. Sur la pratique de la Mercanzia, voir Boschetto, Writing the vernacular.

65 Hayez, Un facteur siennois, pp. 260-261.

66 ASPo, D.1168/1, fol. 55-71, 101v-112, 139v-140, 159-160v, 161v-162 et 184-186v. 
nes de notaires italiens, un passage indique que les écrits marchands ont été traduits par Giottolino di Manfredi, que l'on peut identifier comme un courtier d'épices originaire de Poggibonsi. ${ }^{67}$

Tenor vero dictarum litterarum in ytalico scriptarum per dictum Gerium superius productarum de vulgari in latino redactarum per Joccalinum Mafredi, qui juravit eas fideliter de vulgari in latino redigere, et quas in presenti causa dictus Gerius voluit inseri, sunt prout infra sequitur. ${ }^{68}$

La typologie marchande de l'écrit et peut-être plus précisément la graphie mercantesca des originaux semblait donc prédominer dans le choix du traducteur. Giottolino avait-il une maîtrise plus correcte du latin que la plupart de ses compatriotes marchands de la période ? Les clercs franco-méridionaux furent-ils autant désarmés par ces graphies que par le toscan ? Ou les scribes du même milieu chargés plus tard de copier la procédure pour la remettre au représentant de Bonaccorso firent-il ce travail sans y prêter l'attention nécessaire ? Toujours est-il que de nombreux passages permettent difficilement de restituer le texte original.

Tenor cujusdam lictere scripte in ytalico, que dirigitur Gerio ex parte Bonacursii:

Gheri, Bonachorso, saluto [= salute] di Parigi. Di [= A] 22 dì dy questo ischressi

[= ti scrissi?] e r(ispuosi) ectua [= a tua] lectera qua(n)to bisongnò, e chon essa tenadai [= ti mandai] lo vidim(us) di Chastelecto (e) èe la sopia una (?) della burgesia, la quale èe [= in] mea decta lettere te mandai $\mathrm{p}(\mathrm{er})$ da Mo(n)pul(ieri) Chatalani. Poi a dì 23 d. [= r(icevetti) ?] tua lettre [sic]. R(ispuosi) dì 10 (?) p(er) lo venturieri p(er) chui mandi le mandorle, il quale èe [= àe] facto uno ghrande chorpore [= scalpore] (...). ${ }^{69}$

La Chambre apostolique respectait ainsi la procédure d'inclusion d'écrits fournis par les parties, sans faire une règle absolue de la traduction en latin de langues moins accessibles au personnel local que le provençal ou le français. Si la version destinée aux archives de la Chambre comportait des passages aussi peu compréhensibles que cette copie, on peut s'interroger sur la possibilité de mobiliser ensuite comme preuves des textes aussi incorrects. Le tribunal affirmait sa compétence sur une population d'origine variée évoluant dans un milieu cosmopolite mais ne parvenait pas à réunir les compétences adéquates. Ces passages apparaissent ainsi révélateurs de la difficulté de deux cultures écrites à communiquer ensemble.

67 ADV, Majour, E4, fol. 87, 23v, 179v, etc.; Archivio Apostolico Vaticano, Registra Avenionensia, 204, fol. 459v; ADV, 3 E 12, 244, fol. 34-v.

68 ASPo, D.1168/1, fol. 101v.

69 Ibid., fol. 161v. 
Dans l'accès à la culture juridique, mais aussi à la rhétorique et parfois à la littérature, les notaires jouaient auprès des marchands le rôle essentiel. Sans même compter ses agences catalanes, génoise et pisane, Francesco Datini en avait personnellement employé plusieurs dizaines entre Avignon, Prato et Florence, en privilégiant souvent l'un d'entre eux dans telle ville pendant quelques années, mais en les utilisant aussi en complémentarité dans des rôles diversifiés (connaissance des formules notariales de plusieurs régions, compétences rhétoriques, médiation et relations publiques, relance de débiteurs, etc.) et selon les disponibilités de chacun. ${ }^{70}$ On les retrouve en première position dans les différents secteurs des documents juridiques, tantôt comme producteurs de ces écrits, tantôt comme rhéteurs et chanceliers, tantôt comme interprètes, commentateurs et conseillers.

Dans les deux dernières décennies de la vie de Francesco, son principal médiateur en la matière était ser Lapo Mazzei. Entré en contact direct avec lui en 1390 à l'occasion d'un cycle de vendetta que le marchand voulait apaiser, ser Lapo devint bientôt le principal conseiller de Francesco et l'inspirateur de sa fondation. Ce qui apparaît unique est la polyvalence de ses emplois, qui incluent la direction spirituelle et la fourniture de traduction d'œuvres morales, la connaissance de la classe politique florentine lui permettant d'agir comme conseiller en relations publiques pour protéger du fisc la fortune de son ami, les talents rhétoriques et la connaissance technique du droit pour rédiger des actes valides en diverses circonstances et régions et pour défendre les intérêts du marchand devant des tribunaux. Nous le voyons agir dans tous ces rôles non seulement dans son abondante correspondance (plus de 500 lettres conservées), mais aussi à travers les brouillons qu'il écrivait et les gloses qu'il apposait sur une grande variété de documents, pour orienter précisément l'action de Francesco. ${ }^{71}$ Des dizaines de feuillets conservés portent encore ces gloses du notaire florentin. Dans l'ébauche vernaculaire de testament qu'il élabore pour le marchand, ser Lapo commence par un «pense-bête» destiné à lui-même: il doit se rappeler de prendre la copie du précédent testament, lorsqu'il ira discuter avec Francesco du détail des legs.

70 Les notaires les mieux connus à travers les publications sont ser Lapo Mazzei (Ser Lapo Mazzei, Lettere di un notaro) et ser Bartolomeo Levaldini (Brambilla, Hayez, La maison des fantômes). Pour un premier repérage des notaires les plus employés fondé sur la documentation, voir ibid., pp. 75-87.

71 Les registres et brouillons conservés par ser Lapo contiennent naturellement aussi un certain d'actes rédigés pour Francesco et ses proches (ASFi, Notarile antecosimiano 11492 à 11502) mais c'est lorsqu'il oriente par ses conseils (Ser Lapo Mazzei, Lettere di un notaro, 1, p. 191-192; Brambilla, Hayez, La maison des fantômes, p. 81) ou la communication de formules (voir ci-dessus notes 53 et 60 ) le travail d'un confrère ou du rédacteur d'une scritta, qu'apparaissent mieux ses capacités. 
La glose est alors rédigée en latin: «In rogando, porta tecum librum integri veteris testamenti sui». $^{72}$

Dans un cahier expédié d'Avignon en juin 1405, contenant la procédure latine intentée par Gui de Pesteil contre Francesco en tant que garant d'un autre marchand toscan qui avait contracté une obligation de 3000 florins envers ce chevalier, ser Lapo élabore avec Francesco les réponses que les facteurs d'Avignon devront présenter au tribunal, invoquant entre autres la notion d'usure.

Alla domanda de' f. MMM, Francescho risponde che no, gl'à a dar nulla, come di sotto si mostrerà. E però domanda d'esser finito. ...

Alla domanda di CCC f. l'anno per interessi, dicie Francescho che messer Guido non è di queste cose praticho però che di danari non si dà interessi. ${ }^{73}$

Dans une procédure vernaculaire de la Mercanzia intentée contre Francesco par l'héritière de la famille de trois de ses anciens associés et facteurs, ser Lapo se prononce en revanche sur les points juridiques évoqués, mettant dans la bouche du notaire ou juriste qui va agir comme procureur de Francesco les réponses à invoquer devant le tribunal. Les gloses sont donc rédigées en latin et la première souligne la situation compliquée de Priore di Lorenzo qui, voulant recouvrer les avoirs de son oncle Boninsegna dans la compagnie Datini sans être contraint à assumer les dettes contractées par celui-ci une trentaine d'années plus tôt à l'occasion de sa faillite, n'avait pas formellement assumé la succession de Boninsegna.

Nec fuit heres Prior dicti Boninsegne, qui ante Priorem decessit, nec ista potest adire hereditatem Boninsegne. Et licet Prior in testamento suo dixerit verba quibus demostrat se ut heredem, tamen in testamento hereditas non aditur, sed in vita re ipsa se abstinuit in veritate ab adeundo hereditatem Boninsegne, a quo fuerit institutus, dubitans de infrascriptis creditoribus et cessatione sua quam fecit Florentie et Avinioni, ut infra videbitis. Ideo moriens non curavit dicere in testamento aliqua ex quibus demostrabatur velle se esse heredem. ${ }^{74}$

Dans ces diverses gloses, le choix de la langue lettrée ou vernaculaire apparaît ainsi comme une simple conséquence de la qualité du destinataire du message, lettré ou illetterato, ser Lapo se classant naturellement dans la première catégorie pour se tutoyer.

Cette tentative de cartographie des textes latins contenus dans l'Archivio Datini confirme l'autonomie essentielle de l'écriture marchande par rapport à la

72 ASFi, Notarile antecosimiano, 11502.

73 ASPo, D.1168/4.

74 ASPo, D.1169/2, fol. 9. 
langue de la classe cultivée, jusqu'à la pleine diffusion de l'humanisme. Pour le milieu du négoce, l'accès aux textes latins se limite au mieux à un déchiffrement, plus aisé lorsqu'il s’agit de documents accoutumés qui ont simplement revêtu une forme latine que lorsqu'on change complètement de registre linguistique. Ce survol confirme néanmoins aussi l'habitude des marchands de se procurer, par goût ou par nécessité, des textes variés étrangers à leur culture, qui pour certains relevaient d'une veine littéraire mais pour la plupart s'inscrivaient dans un contexte juridique. Assez loin des formes de traduction littéraire mises en évidence par l'étude des volgarizzamenti, les modes de communication entre marchands appartenant à différentes nations ou les traductions incertaines du tribunal de la Chambre apostolique révèlent en outre une dimension évidente de bricolage, de copie passive de textes qui ne paraissent même pas toujours interprétés, plus qu'une pratique théorisée et développée selon des normes rigoureuses. Dans ce panorama, un secteur nous reste peu accessible pour reconstruire un cadre plus complet des contacts entre latin et vernaculaires. C'est celui qui éclairerait mieux la coexistence en un lieu ou une société urbaine de la langue des lettrés avec le «latin vernaculaire» des marchands génois, piémontais et lombards. La pratique courante de celui-ci leur donnait-elle une capacité majeure à lire des actes notariés ou des œuvres littéraires composées en cette langue? Il n'existe certes pas d'équivalent génois ou milanais du fonds Datini; ni même, semble-t-il, de fonds épistolaire important qui pourrait refléter une diversité sociale marquée parmi les expéditeurs, ou de série abondante de livres de comptes et de famille. La présence mineure dans l'Italie du nord des volgarizzamenti littéraires, religieux et administratifs (comme les statuts d'institutions), tend néanmoins à suggérer que dans cette aire culturelle la catégorie des semi-lettrés pouvait peut-être se contenter d'écouter et de lire des textes latins.

Pour revenir en Toscane, dans les contextes où la précision sémantique et les compétences culturelles apparaissent plus importantes, le notaire fait figure de passeur privilégié. La polyvalence des rôles que revêtent certains d'entre eux comme Mazzei fait également penser à d'autres figures de truchements privilégiés dans la sphère plus typique de l'activité marchande. Dans les régions arabes, turques et persanes, le turciman ou dragoman n'œuvrait pas seulement comme interprète; il était aussi souvent un guide, parfois un hôte, assurément un courtier pour introduire l'étranger sur d'autres places fréquentées par les marchands, à peu près comme le «fixeur» d'un reporter moderne. Dans diverses situations liées à la culture lettrée, le notaire était l'auxiliaire indispensable du marchand comme l'était l'hôte ou le courtier dans la sphère logistique. Mais la connaissance du latin administratif-juridique de base et des formules nécessaires pour rédiger une quittance ou la vente d'une terre étaient certainement plus 
diffuses que la capacité à établir un acte destiné à être produit dans un pays éloigné, comme une procuration, ou qui devait respecter des normes statutaires de deux aires différentes, comme un contrat de mariage ou un testament impliquant comme parties des étrangers. Tous ces notaires n'étaient pas non plus dotés d'ascendant moral, de sens politique ou de la connaissance intime des notables influents du cru. Pour un poste de facteur dans une agence, les marchands ne considéraient pas comme critère premier la capacité des candidats à s'exprimer dans des langues secondes, par rapport à la maîtrise des formes de l'écrit marchand. La connaissance du latin devait de la même façon leur sembler un élément implicite, et non la pierre de touche, pour évaluer le meilleur passeur de la langue du droit ou de la rhétorique, en comparaison de compétences plus techniques. 
